\title{
Downregulation of hemojuvelin prevents inhibitory effects of bone morphogenetic proteins on iron metabolism in hepatocellular carcinoma
}

\author{
Ulrike Maegdefrau ${ }^{1}$, Stephanie Arndt ${ }^{1}$, Georgi Kivorski ${ }^{2}$, Claus Hellerbrand ${ }^{2}$ and Anja-Katrin Bosserhoff ${ }^{1}$
}

Recently, we revealed that bone morphogenetic protein (BMP) 4 is increased in hepatocellular carcinoma (HCC). Furthermore, latest reports described BMPs, in particular BMP6, as important regulators of hepcidin expression in iron homeostasis. Therefore, we aimed to unravel why enhanced BMP expression in HCC patients does not lead to severe changes in iron metabolism. Initial analysis of the BMP4 and BMP6 expression patterns revealed enhanced expression on mRNA and protein level in HCC cell lines and tissue samples compared with primary human hepatocytes (PHHs) and normal liver tissues. However and interestingly, hepcidin expression was reduced in HCC cell lines and tissues. Analysis of BMP6 receptor expression revealed loss of BMP6-specific receptor subunit in HCC. To identify a possible regulatory mechanism causing lack of reaction to BMP4 we analyzed the expression of hemojuvelin (HJV), which is involved in iron metabolism as BMP co-receptor. HJV expression was markedly decreased in HCC cell lines and tissues. HJV promoter analysis revealed potential HNF- $1 \alpha$ and snail-binding sites, but functional analysis ruled out that these transcriptional regulators or promoter methylation are the cause of HJV downregulation in HCC. However, we identified AU-rich elements in the HJV $3^{\prime}$-untranslated region and revealed significantly faster decay of HJV mRNA in HCC cells as compared with PHH indicating decreased mRNA-stability as the reason for the loss of HJV expression in HCC.

Laboratory Investigation (2011) 91, 1615-1623; doi:10.1038/labinvest.2011.123; published online 22 August 2011

KEYWORDS: bone morphogenetic protein 4 and 6; hemojuvelin; hepatocellular carcinoma; hepcidin; iron metabolism

In the last few years, our knowledge of the regulation of iron homeostasis significantly increased. ${ }^{1}$ Thus, bone morphogenetic proteins (BMPs) have been newly identified as regulators of hepatic hepcidin expression. ${ }^{2-5} \mathrm{HJV}$ (hemojuvelin), a member of the repulsive guidance molecule family (RGM family), acts as a BMP co-receptor and triggers the binding of BMP ligands to BMP receptors to enhance hepcidin expression. ${ }^{3,4}$ For a long time BMP2, 4, 6 and 9 were postulated as important regulators of hepcidin expression in vitro, ${ }^{6,7}$ whereas in latest publications along with others, we described especially BMP6 as key endogenous regulator of hepcidin expression and iron metabolism in vivo. ${ }^{6,8-10}$

It is furthermore known that BMPs not only have an important role in the iron sensing pathway but are also involved in the development and progression of different kinds of cancer. ${ }^{1-13}$ We recently demonstrated that BMP4 promotes the progression of hepatocellular carcinoma (HCC). ${ }^{14}$ Noteworthy, up to now the impact of enhanced
BMP expression on (changes in) iron metabolism in HCC, one of the most common cancers worldwide, ${ }^{15}$ has not been addressed. Thus, one may hypothesize that patients with enhanced BMP expression levels develop anemia through increased levels of hepcidin, which is responsible for internalization and degradation of ferroportin to limit intestinal iron uptake. ${ }^{16,17}$ However, along with others, we found increased iron levels in the serum of HCC patients. ${ }^{18,19}$ Therefore, we aimed to unravel the reason why HCC patients with enhanced BMP expression levels do not come down with severe changes in iron metabolism.

To answer this question, we first analyzed whether the expression of BMP6, the main regulator of iron metabolism, is also enhanced in HCC. Next, we investigated the expression of hepcidin and the influence of different modulators involved in the BMP-Smad signaling pathway, respectively, because overall the mechanisms, which regulate iron metabolism in HCC are largely unknown.

\footnotetext{
${ }^{1}$ Institute of Pathology, University of Regensburg, Regensburg, Germany and ${ }^{2}$ Department of Internal Medicine I, University of Regensburg, Regensburg, Germany Correspondence: Dr A-K Bosserhoff, PhD, Institute of Pathology, University of Regensburg, Franz-Josef-Strauss-Allee 11, D-93053 Regensburg, Germany.

E-mail: anja.bosserhoff@klinik.uni-regensburg.de

Received 13 December 2010; revised 24 June 2011; accepted 26 June 2011
} 


\section{MATERIALS AND METHODS Cells and Cell Culture}

Human HCC cell lines Hep3B (ATCC HB-8064) and PLC (ATCC CRL-8024) were cultured as described. ${ }^{20}$ Primary human hepatocytes (PHHs) were freshly isolated and cultured as previously described. ${ }^{21}$ Human liver tissue for cell isolation was obtained according to the guidelines of the charitable state-controlled foundation HTCR with the patient's informed consent.

\section{Human Tissues and Serum Samples}

Primary HCC tissues were obtained from HCC patients undergoing surgical resection. Histologically confirmed normal liver tissues served as control. Further, serum samples of HCC and control patients were collected. Tissue as well as serum samples were processed without delay, for example, immediately snap frozen and stored at $-80^{\circ} \mathrm{C}$. Informed consent was obtained from all patients, and the study was approved by the local ethics committee.

\section{Immunohistochemistry}

Paraffin-embedded preparations of normal liver and HCC tissues were screened for HJV protein expression. The tissues were deparaffinized, rehydrated and subsequently heated in a pressure cooker for $5 \mathrm{~min}\left(120^{\circ} \mathrm{C}\right)$ in Tris/EDTA buffer ( $\mathrm{pH}$ 9.0). After blocking with $\mathrm{H}_{2} \mathrm{O}_{2}$, tissue sections were incubated for 30 min with goat anti-HJV (AF-3720) antibody (1:20; R\&D Systems, Minneapolis, MN, USA) or goat antiBMP6 (S-20; sc-27408) antibody (1:50; Santa Cruz Biotechnology, Heidelberg, Germany) in antibody diluent with background reducing components (DakoCytomation, Dako North America, Carpinteria, CA, USA). The secondary PODconjugated anti-goat antibody (Sigma-Aldrich, Munich, Germany) was incubated for $30 \mathrm{~min}$ at room temperature. Antibody binding was visualized using $\mathrm{DAB}+$ solution (DakoCytomation), and counterstaining was performed with haemalaun solution (DakoCytomation). Evaluation of the staining was performed semi-quantitatively by light-microscopy (AxioVision, Zeiss, Munich, Germany).

\section{RNA Isolation and Reverse Transcription}

Total cellular RNA was isolated from cultured cells and tissue samples using a RNeasy kit (Qiagen, Hilden, Germany), and cDNAs were generated as previously described. ${ }^{11}$

\section{Analysis of mRNA Expression by Quantitative PCR}

Total mRNA expression was analyzed by quantitative realtime PCR with specific sets of primers (Table 1) applying LightCycler technology (Roche, Mannheim, Germany) as described. ${ }^{11,22}$ Gene expression levels were normalized to $\beta$-actin. Moreover, hepcidin expression levels of $\mathrm{PHH}$ and HCC cells after BMP4 treatment were exemplarily normalized to a set of additional housekeeping genes (GAPDH, HPRT, SFRS4) to rule out side effects caused by regulation of the housekeeper $\beta$-actin. No variations in the expression levels were observed (data not shown). ${ }^{23}$

\section{Stimulation of PHH and HCC Cells}

Cells were seeded into six-well plates $\left(2 \times 10^{5}\right.$ HCC cells/ $1 \times 10^{6}$ PHH cells per well) and were treated with $50 \mathrm{ng} / \mathrm{ml}$ recombinant BMP4 (R\&D Systems) and $150 \mathrm{ng} / \mathrm{ml}$ noggin (R\&D Systems), respectively, for $20 \mathrm{~h}$ in normal High Glucose DMEM (PAN Biotech GmbH, Aidenbach, Germany) without FCS. Subsequently, mRNA was extracted as indicated above.

For mRNA stability analysis, actinomycin D $(7.5 \mu \mathrm{g} / \mathrm{ml}$; Sigma-Aldrich) was added to the medium and mRNA was isolated after 2, 4 and $6 \mathrm{~h}$. Subsequently, qRT-PCR was performed to analyze mRNA expression and half-life of HJV mRNA was evaluated by one phase exponential decay of nonlinear regression using GraphPad Prism Software (GraphPad Software, San Diego, CA, USA). All treatments were repeated at least three times.

\section{Transfection Experiments}

Flag-tagged human HJV expression plasmid was a generous gift from Herbert Y Lin (Harvard Medical School, Boston, MA, USA). ${ }^{3}$ HNF- $1 \alpha$ and antisense snail (as-snail) expression constructs were previously described. ${ }^{24,25}$ For transient transfections, PLC cells $\left(2 \times 10^{5}\right.$ per well $)$ were seeded into

Table 1 Primers used for quantitative RT-PCR analysis

\begin{tabular}{|c|c|c|}
\hline \multirow[t]{2}{*}{ Name } & \multicolumn{2}{|c|}{ Nucleotide sequence } \\
\hline & Forward primer & Reverse primer \\
\hline$\beta$-Actin & 5'-CTACGTGGCCCTGGACTTCGAGC-3' & 5'-GATGGAGCCGCCGATCCACACGG-3' \\
\hline BMP6 & 5'-AAGGCTGGCTGGAATTGACATCACG-3' & 5'-GGTAGAGCGATTACGACTCTGTTGTC-3' \\
\hline Alk2 & 5'-GCCTGGAGCATTGGTAAGC-3' & 5'-CTGCCCACAGTCCTTCAAG-3' \\
\hline TFR2 & 5'-AACCCAGTTCCCTCCAGTTG-3' & 5'-GCAGCCGAAGATGTTGTTGA-3' \\
\hline
\end{tabular}


six-well plates and were transfected with $0.01 \mu \mathrm{g}(\mathrm{HJV}) / 0.5 \mu \mathrm{g}$ (HNF-1 $\alpha /$ as-snail) expression plasmid using the Lipofectamine Plus method (Invitrogen, Carlsbad, CA, USA) according to the manufacturer's instructions (transfection efficiency was approximately 40\%). Subsequent experiments were performed $20 \mathrm{~h}$ after transfection. All transfection experiments were repeated at least three times.

\section{Western Blot Analysis}

Protein extracts of cells were prepared as described. ${ }^{11}$ Protein extracts from normal and HCC tissues were homogenized in $200 \mu \mathrm{l}$ RIPA-buffer (Roche). Insoluble fragments were removed by centrifugation at 13000 r.p.m. for $10 \mathrm{~min}$, and the supernatant was frozen and stored at $-80^{\circ} \mathrm{C}$. For western blotting, protein lysates were separated on SDS-PAGE gels and blotted onto a PVDF membrane. After blocking with BSA/TBS, primary antibodies were applied (anti-BMP6 (S-20); 1:500, Santa Cruz Biotechnology; anti-HJV, 1:2000, $\mathrm{R} \& \mathrm{D}$ Systems). Alkaline phosphatase-conjugated anti-goat antibody (1:5000, Chemicon, CA, USA) or anti-mouse antibody (1:4000, Chemicon) served as secondary antibodies.

\section{Enzyme-Linked Immunosorbent Assay (ELISA)}

Serum samples were analyzed by an ELISA to quantify hepcidin pro-hormone serum levels (DRG Instruments, Marburg, Germany) according to the manufacturer's instructions.

\section{Statistical Analysis}

Statistical analyses were performed using SPSS version 10.0 (SPSS, Chicago, IL, USA) and GraphPad Prism Software. Results are expressed as mean \pm s.e.m. (range) or percent. Comparison between groups was made using Student's unpaired $t$-test. A $P$-value $<0.05$ was considered as significant. Power analysis was performed using PASS-11 for qRT-PCR experiments with small sample sizes. Numbers of analyzed samples (HCC and normal liver tissues) were adapted for each gene individually to ensure sufficient power values.

\section{RESULTS \\ BMP6 Expression in HCC}

Previously, we have shown enhanced BMP4 expression in HCC. ${ }^{14}$ Here, we further detected increased BMP6 mRNA expression in HCC cell lines (Hep3B and PLC cells) compared with PHHs (Figure 1a). Western blot analysis

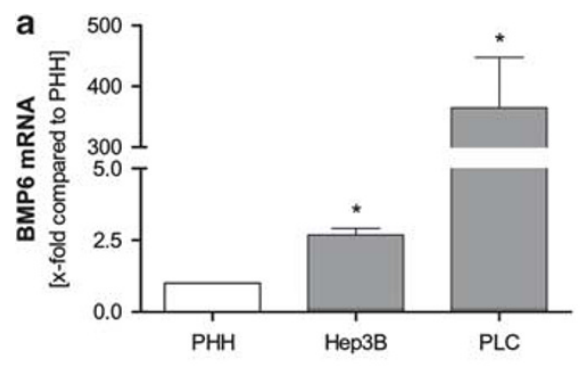

d
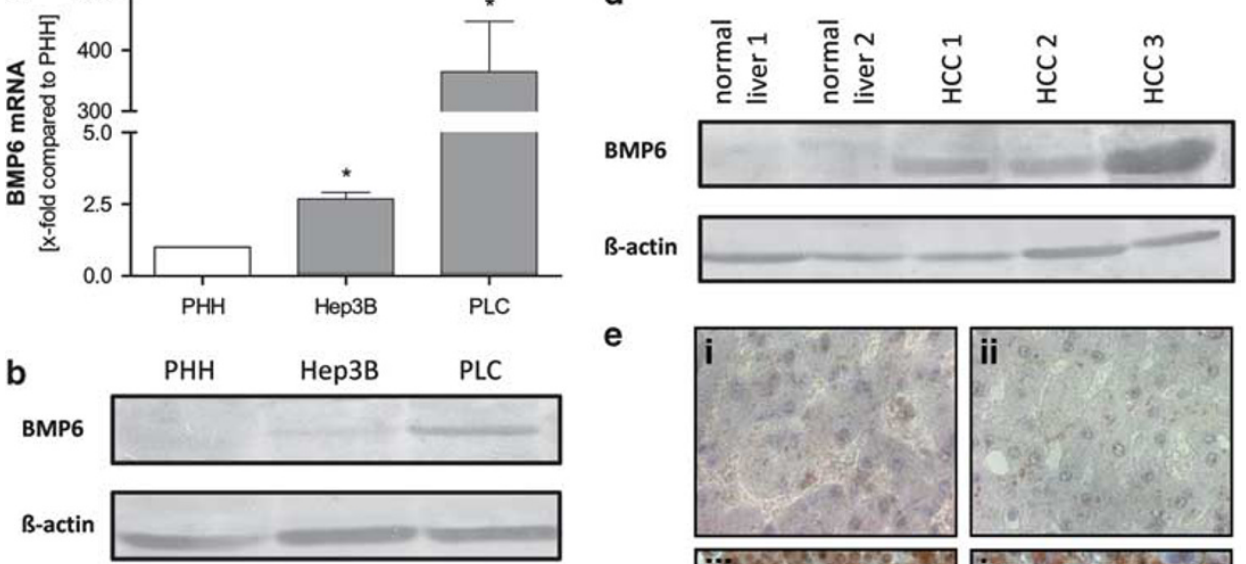

c
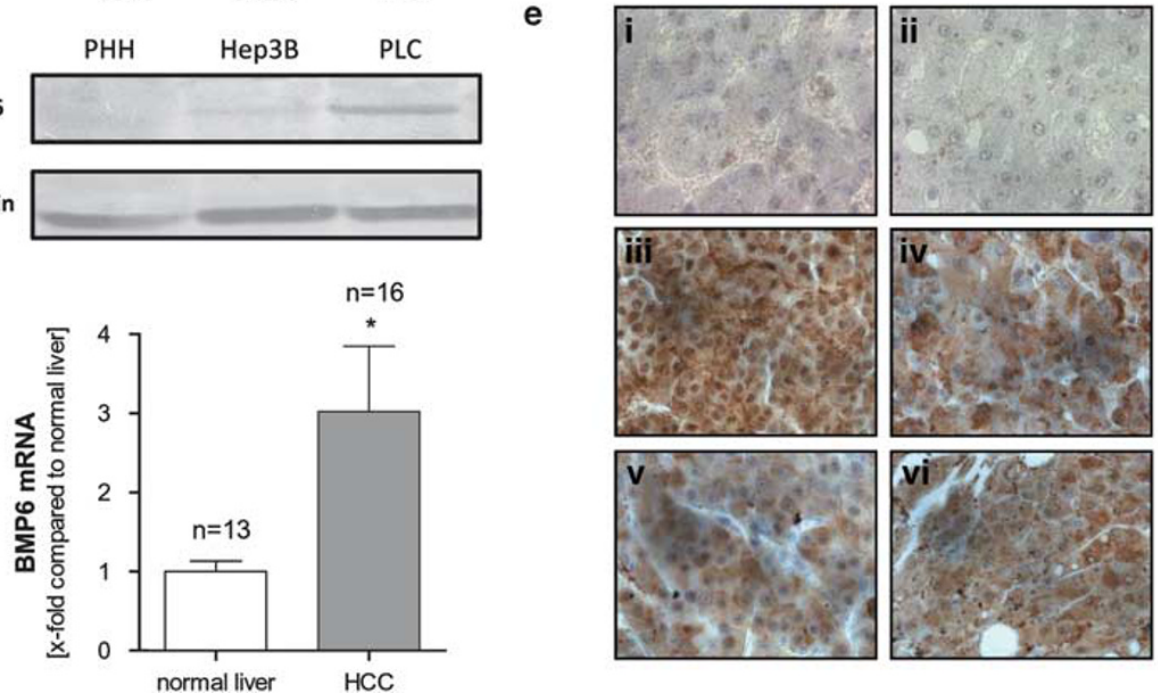

Figure 1 Expression of BMP6 in HCC. (a) BMP6 mRNA expression was analyzed by qRT-PCR. HCC cell lines showed increased BMP6 levels compared with primary human hepatocytes $(\mathrm{PHH}) .\left({ }^{*} P<0.05\right.$ compared with $\left.\mathrm{PHH} ; \mathrm{PHH}=1\right)$. (b) Western blot data confirmed enhanced expression levels of BMP6 in HCC cell lines PLC and Hep3B. $\beta$-Actin serves as loading control. (c, d) Analysis of 13 human normal liver tissues compared with 16 HCC tissues by qRT-PCR (c) and western blot (d) revealed enhanced BMP6 levels in HCC tissues in vivo. In the western blot, exemplary two normal liver tissues compared with three HCC tissues are shown. $\left({ }^{\star} P<0.05\right.$; normal liver $\left.=1\right)$. (e) Immunohistochemical stainings of normal liver tissues and HCC tissues revealed strong BMP6 expression in HCC tissues as shown in representative pictures of four HCC tissues (iii, iv, $\mathbf{v}$ and vi) compared with two normal liver tissues (i $+\mathbf{i i})$ (magnification $\times 40$ ). 
confirmed the upregulation of BMP6 in HCC cell lines on the protein level (Figure 1b).

In accordance, quantitative RT-PCR and western blot analysis revealed enhanced BMP6 expression in HCC tissues compared with non-tumorous liver tissues (Figures $1 \mathrm{c}$ and $\mathrm{d}$ ). Immunohistochemistry confirmed enhanced BMP6 expression in HCC tissues compared with non-tumorous liver tissues (Figure 1e).

\section{Influence of BMP4 and BMP6 on Hepcidin Expression in HCC}

Both BMP4 and BMP6 affect iron levels through regulation of hepcidin expression. ${ }^{4,6-8}$ Thus, one might have expected that increased BMP expression in HCC leads to increased hepcidin expression levels and decreased serum iron levels, respectively. However, analysis of HCC patients revealed slightly increased serum iron levels compared with the iron levels of control patients (Supplementary Figure 1A). Further, serum transferrin saturation and ferritin levels were enhanced in HCC patients (Supplementary Figures 1B and C). Strikingly, also hepcidin expression was reduced in HCC cell lines and tissues as compared with $\mathrm{PHH}$ and non-tumorous liver tissues, respectively (Figures $2 \mathrm{a}$ and $\mathrm{b}$ ). Furthermore, HCC patients revealed significantly lower hepcidin serum levels than controls (Figure 2c).

In search for a possible cause for weak hepcidin expression (despite high BMP levels) in HCC, we evaluated Alk2 expression in HCC. Previous studies revealed this BMPreceptor as critical for the regulation of hepcidin by BMP6, ${ }^{5}$ which is known to be the main regulator of iron homeostasis under physiological conditions. ${ }^{6,8}$ Noteworthy, Alk2 expression was significantly decreased in HCC cell lines and HCC tissues (Figures $3 \mathrm{a}$ and $\mathrm{b}$ ).

Besides BMP6, BMP4 has been described as important regulator of hepcidin expression. ${ }^{4,6-8}$ However and interestingly, BMP4 stimulation ( $50 \mathrm{ng} / \mathrm{ml} ; 20 \mathrm{~h}$ ) exhibited almost no (PLC) or significantly weaker effects (Hep3B), respectively, on hepcidin expression in HCC cells compared with $\mathrm{PHH}$ (Figure 3c).
Here, it was conceivable that loss of Alk3 receptor expression would be responsible for reduced hepcidin induction in HCC, because Alk3 is the most important BMP receptor subunit to mediate BMP4 signaling. ${ }^{26}$ To rule out potential effect of loss of Alk3 in HCC, we analyzed Alk3 expression levels by qRT-PCR in HCC cell lines and tissues compared with $\mathrm{PHH}$ and normal liver tissues, respectively. Neither in HCC cell lines nor in HCC tissues reduced Alk3 receptor expression levels could be detected (data not shown). Furthermore, we analyzed expression of transferrin receptor 2 (TFR2), which is known to be involved in hepcidin regulation. ${ }^{27,28}$ qRT-PCR analysis of TFR2 displayed reduced expression levels of TFR2 in HCC tissues and cell lines compared with normal liver tissues or $\mathrm{PHH}$, respectively (Supplementary Figures 1A and B).

To further identify the reason for the diminished BMP effects on hepcidin expression in HCC, we analyzed HJV, because it is well described that HJV acts as a BMP coreceptor, and hereby, enhances BMP-induced hepcidin expression. ${ }^{5}$ Importantly, we detected markedly reduced HJV mRNA (Figure 4a) and protein (Figure $4 \mathrm{~b}$ ) expression in HCC cell lines compared with PHH. In accordance, HJV was significantly downregulated in human HCC tissues (Figures $4 \mathrm{c}$ and d). In situ analysis of HJV by immunohistochemistry confirmed strong membrane staining in normal liver tissues but no detectable immunosignal in HCC tissues. Representative pictures are shown in Figure 4e together with immunostainings of positive (heart) and negative (salivary glands) tissue controls.

To determine whether the loss of HJV expression is responsible for the reduced BMP4 effects on hepcidin expression in HCC cells, PLC cells were transfected with a full-length HJV expression construct (Figure 5a). HJV expression did not affect basal hepcidin expression in HCC cells (Figure 5b). However, BMP4-induced hepcidin expression was significantly higher in PLC cells transfected with the HJV expression plasmid compared with control cells transfected with the empty vector (Figure $5 b$ ). To ensure that induction of hepcidin expression in HJV transfected cells was actually mediated by BMP4, we treated HJV and control
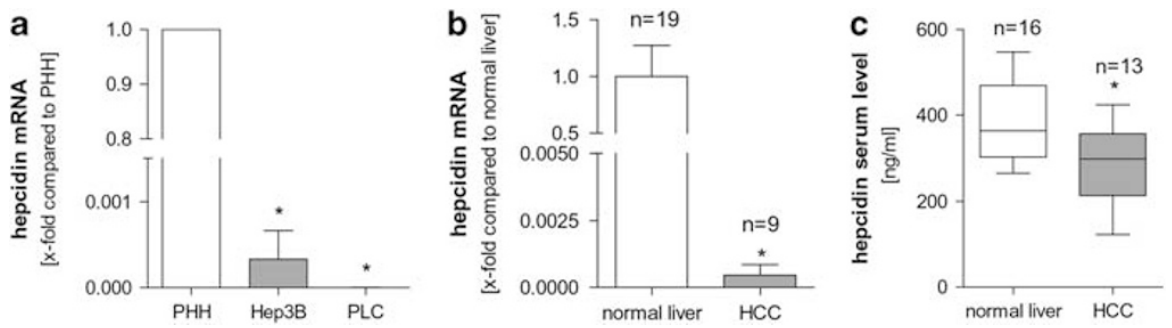

Figure 2 Expression of hepcidin in HCC. $(\mathbf{a}, \mathbf{b})$ Analysis of mRNA by qRT-PCR showed reduced hepcidin expression levels in HCC cell lines $\left({ }^{*} P<0.05\right.$ compared with $\mathrm{PHH} ; \mathrm{PHH}=1)(\mathbf{a})$. Nine analyzed human $\mathrm{HCC}$ tissues confirmed the results, expression was lower in the HCC tissues compared with 19 normal liver tissues $\left({ }^{*} P<0.05\right.$; normal liver $\left.=1\right)(\mathbf{b})$. (c) To determine the hepcidin pro-hormone protein concentration we performed an ELISA assay. Measurement of 16 serum samples of healthy patients compared with 13 serum samples of HCC patients revealed decreased hepcidin levels in HCC patients $\left({ }^{*}<<0.05\right)$. 

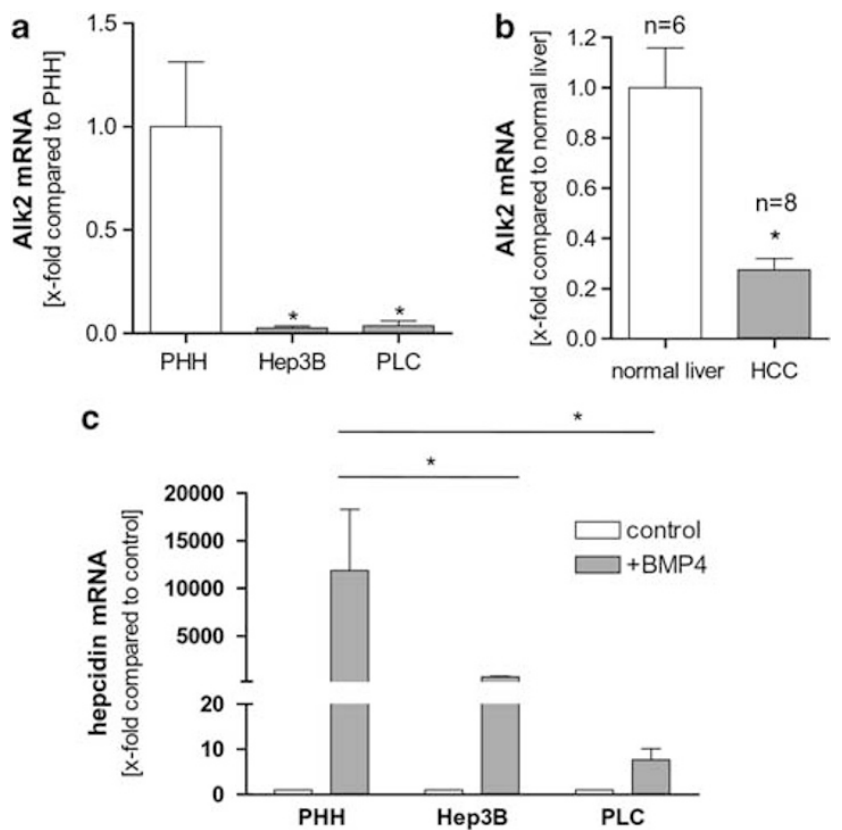

Figure 3 Influence of BMP4 and BMP6 expression on hepcidin levels. (a, b) To determine whether BMP6 is able to mediate effects on hepcidin expression, we performed qRT-PCRs of BMP6 specific type I receptor subunit (Alk2). Quantitative RT-PCR revealed downregulation of Alk2 expression in vitro in the HCC cell lines $\left({ }^{*} P<0.05\right.$ compared with $\mathrm{PHH}$; $\mathrm{PHH}=1)(\mathbf{a})$ and in vivo in eight $\mathrm{HCC}$ tissues $\left({ }^{*} P<0.05\right.$; normal liver $\left.=1\right)(\mathbf{b})$. (c) To analyze induction of hepcidin expression after BMP4 treatment, we stimulate PHH cells and HCC cells with $50 \mathrm{ng} / \mathrm{ml} \mathrm{BMP4}$ for $20 \mathrm{~h}$ and evaluate the mRNA levels. Effects by treatment with BMP4 on hepcidin expression were much stronger in PHHs compared with HCC cell lines. $\left({ }^{*} P<0.05\right.$ compared with $\mathrm{PHH}$; control $=1$ ).

transfected HCC cells with noggin together with BMP4. As expected, we observed inhibition of BMP4-induced hepcidin induction in HJV transfected PLC cells after noggin treatment (data not shown).

These findings suggest that the loss of HJV mainly causes the lack of effects of enhanced BMP expression on hepcidin expression in HCC.

\section{Potential Mechanism Leading to HJV Deficiency in HCC}

To investigate potential mechanisms of HJV regulation, we performed in silico promoter studies with the program Gene2Promotor (Genomatix, AnnArbor, MI, USA) and identified binding sites of the transcription factors HNF- $1 \alpha$ and Snail in the HJV promoter region (position -63 to -39 , -674 to -654 , and -38 to -18 , respectively, to the transcription start site). HNF-1 $\alpha$ is known to be a tumor suppressor, which is lost in HCC. ${ }^{20}$ To analyze effects of HNF-1 $\alpha$ on the expression of HJV in HCC cells, we transfected Hep3B and PLC cells with an HNF- $1 \alpha$ expression vector. HNF- $1 \alpha$ transfection for $24 \mathrm{~h}$ (data not shown) or $48 \mathrm{~h}$ (Figure 6a) did not affect HJV expression in HCC cells.

Next, we were interested in potential effects of Snail, a transcriptional repressor strongly expressed in HCC. ${ }^{29}$
However, also transfection with an as-snail construct for 24 or $48 \mathrm{~h}$ did not change HJV expression levels in Hep3B and PLC cells (data not shown).

Furthermore, no CpG islands could be detected in the putative $H J V$ promoter region, thus ruling out a potential influence of promoter hypermethylation on HJV regulation. However, analysis of the $3^{\prime}$-untranslated region indicated AUrich regions (at mRNA position 1841, 1868 and $1931 \mathrm{bp}$ ), which hints to mRNA stability as a possible mechanism of HJV regulation in HCC. To analyze mRNA stability, PHH and HCC cells were treated with actinomycin D $(7.5 \mu \mathrm{g} / \mathrm{ml})$ for 2,4 and $6 \mathrm{~h}$ to inhibit transcription of mRNA. Interestingly, qRT-PCR analysis revealed a significantly faster decay of HJV mRNA in Hep3B and PLC cells compared with PHH (Figure 6b).

Taken together, these data suggest that reduced HJV expression mediated by mRNA stability is an important mechanism to compensate effects of enhanced BMP expression in HCC.

\section{DISCUSSION}

Enhanced BMP expression leads to increased migration, invasion and angiogenesis of tumor cells, and thereby, BMPs display an important role for tumor progression. ${ }^{11,14,22}$ However, BMPs are also involved in regulation of iron metabolism. Especially BMP6 is described as main regulator of iron metabolism in vivo by increasing hepatic hepcidin expression. ${ }^{6,8-10}$ Therefore, we analyzed BMP6 expression in HCC and found, that in addition to BMP4, ${ }^{14,15}$ also BMP6 expression was enhanced in HCC.

These findings suggested that hepcidin levels should be increased in HCC. Results on the expression of hepcidin in HCC reported in former studies were not completely conclusive. Thus, it has been described that hepcidin mRNA expression is uniformly suppressed in HCC, whereas serum concentration of hepcidin was not correlated with mRNA expression in these studies. ${ }^{30-32}$ However, up to now the influence of enhanced BMP levels of hepcidin expression in HCC was not analyzed. Remarkably, in spite of enhanced BMP expression in HCC, we were able to detect reduced hepcidin mRNA and protein expression levels in HCC tissues and cells.

To identify a possible reason for the reduced hepcidin levels in HCC, we analyzed expression pattern of the BMP6specific receptor. BMP6 mediates its effects via binding to the BMP type I receptor (Alk2 or Alk3), which forms a complex with BMP type II receptor (ActRIIA) and HJV. This leads to phosphorylation of Smad 1,5 and 8, which translocate in complex with Smad4 into the nucleus and activate hepcidin expression. ${ }^{5,33}$ It is well described that BMP2 and BMP4 bind to BMP type I receptor subunits Alk3 and Alk6 to enhance target gene expression. However, Alk6 is not expressed in the liver and BMP6 binds additionally to Alk2. ${ }^{5,26}$ Thus, we analyzed BMP6-specific type I receptor expression and observed strong expression of Alk2 in PHH cells and normal 

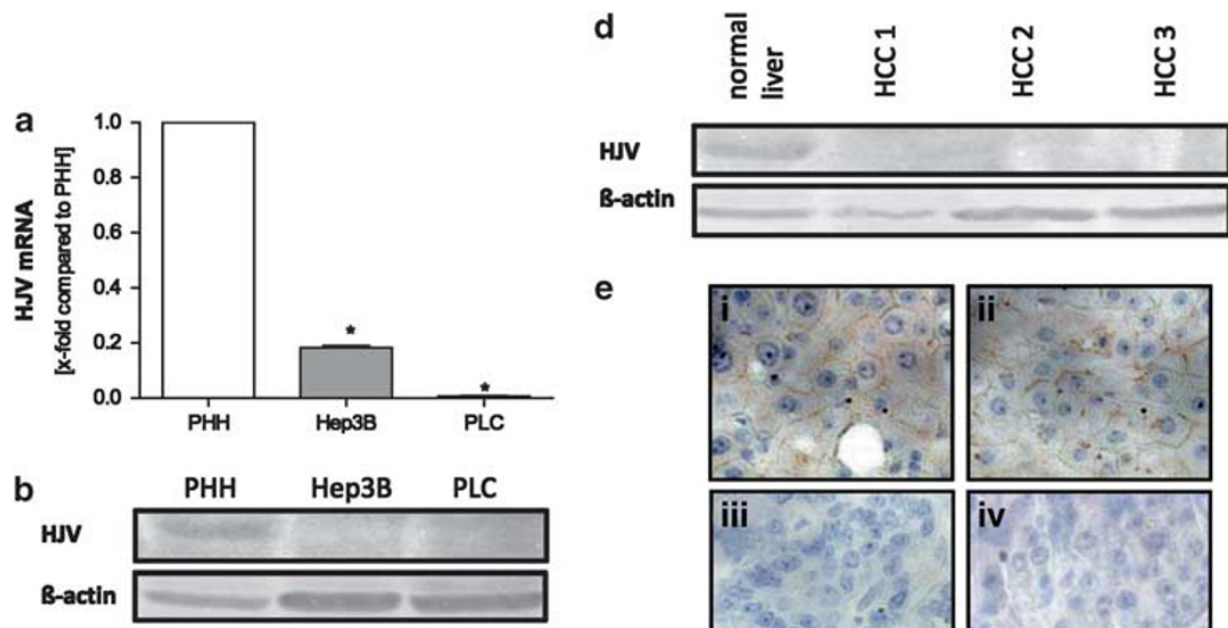

e
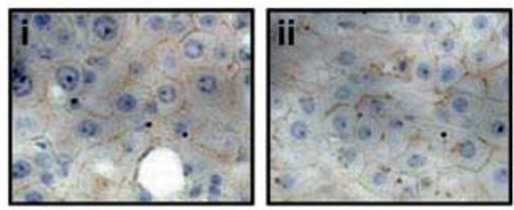

C
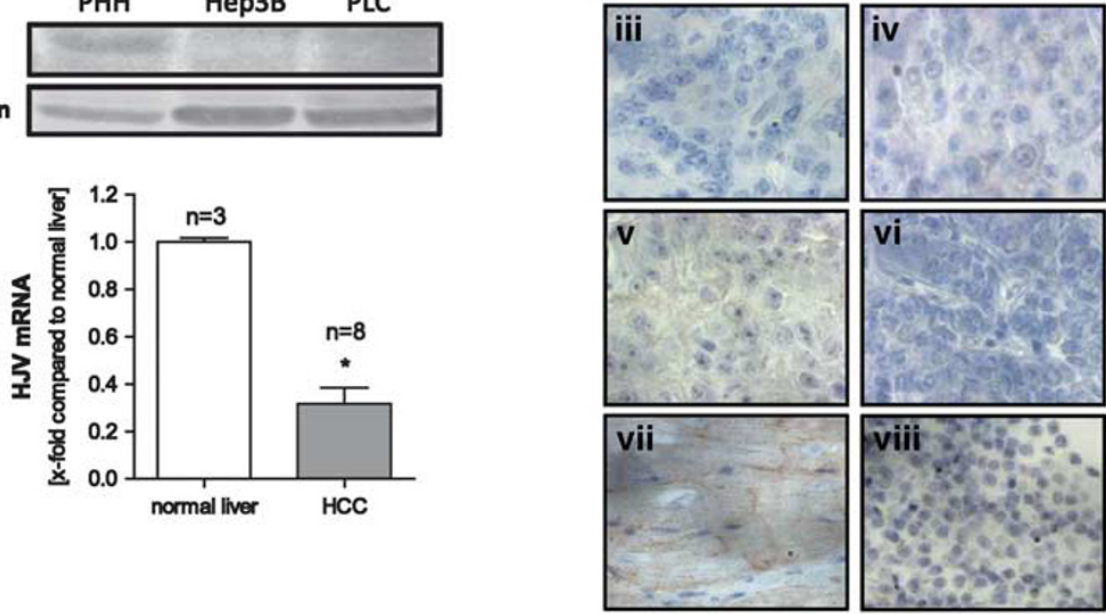

Figure 4 Expression of HJV in HCC. (a, b) Investigations of mRNA (a) and protein levels (b) of HJV revealed strongly reduced HJV levels in $\mathrm{HCC}$ cell lines compared with PHHs. $\beta$-Actin serves in western blot analysis as loading control. $\left({ }^{*} P<0.05\right.$ compared with $\left.\mathrm{PHH} ; \mathrm{PHH}=1\right)$. (c, d) Expression of HJV mRNA (c) was also reduced in eight HCC tissues compared with three normal liver tissues. This was confirmed on protein level performing western blot as exemplified by three HCC tissue lysates shown in $(\mathbf{d})$. $\left({ }^{*} P<0.05\right.$; normal liver $\left.=1\right)$. (e) HJV protein expression was further analyzed by immunohistochemistry, shown in representative pictures of two normal liver tissues (i $+\mathbf{i i})$ and four HCC tissues (iii, iv, $\mathbf{v}$ and $\mathbf{v i}$ ). Strong membrane staining of HJV was detectable in normal liver tissues whereas HCC tissues showed only weak staining (magnification $\times 40$ ). Sections of heart tissue (vii) and salivary gland (viii) serves as positive and negative controls, respectively.
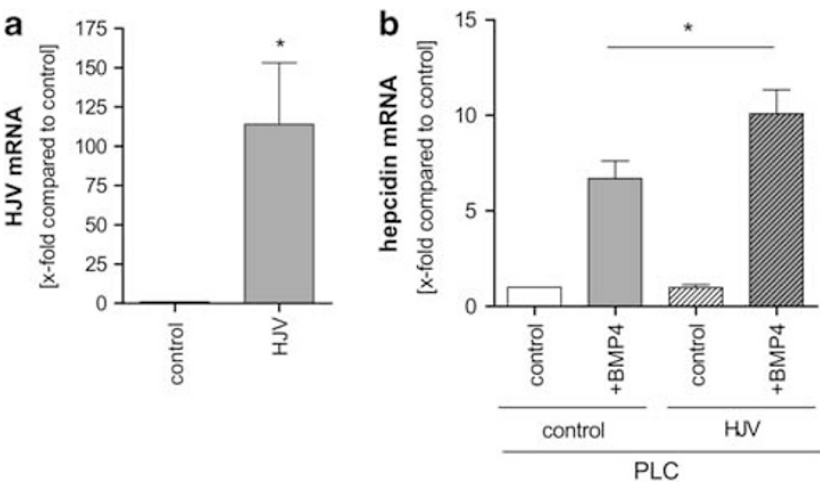

Figure 5 HJV as modulator of hepcidin expression. (a) HJV mRNA expression was analyzed in PLC cells transfected with HJV expression construct compared with control transfected cells (pCMX-PL2-Flag). HJV mRNA expression was enhanced in HJV transfected cells compared with control transfected cells. $\left({ }^{*} P<0.05\right.$; control $\left.=1\right)$. (b) Induction of hepcidin mRNA expression was much stronger in HJV transfected PLC cells after BMP4 stimulation for $20 \mathrm{~h}$ compared with control transfected cells after BMP4 stimulation. $\left({ }^{*} P<0.05\right.$ BMP4 treated HJV transfected cells compared with BMP4 treated control transfected cells; control $=1$ ).
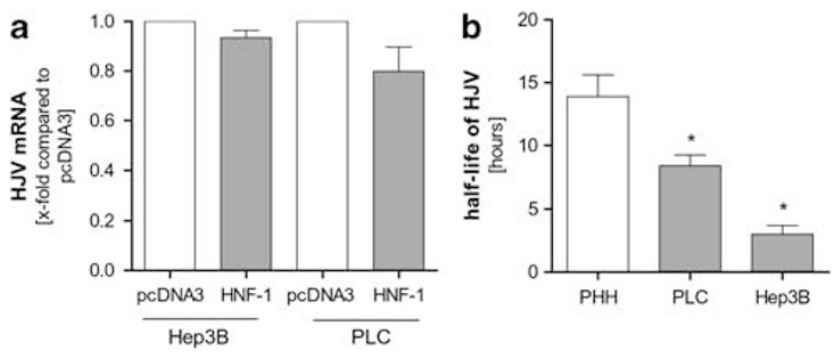

Figure 6 Potential mechanisms leading to HJV deficiency. (a) HJV promoter studies revealed binding sites for HNF-1 $\alpha$ (HNF-1). Transfection experiments of HCC cells with HNF-1 $\alpha$ full-length construct for 24 and $48 \mathrm{~h}$ ( $48 \mathrm{~h}$ values are illustrated) showed no changes of HJV mRNA expression pattern compared with control transfected cells (pcDNA3); (pcDNA3 $=1)$. (b) To analyze RNA stability of HJV in HCC cell lines compared with $\mathrm{PHH}$ cells we stimulated PLC, Hep3B and PHH cells with actinomycin D $(7.5 \mu \mathrm{g} / \mathrm{ml})$ and incubation was continued for 2,4 and $6 \mathrm{~h}$. HJV mRNA expression in the cells was analyzed by qRT-PCR for each timepoint and half-life of HJV was evaluated with GraphPad Prism Software. HCC cell lines showed a significantly reduced half-life of HJV compared with $\mathrm{PHH}$. $\left({ }^{*} P<0.05\right)$. 
liver tissues, whereas expression is lost in HCC cells and HCC tissues. This finding explains why BMP6 is not able to induce hepcidin expression in HCC.

Besides BMP6, different BMPs were discussed for regulation of iron metabolism in vitro, where BMP4 was mainly described as valid regulator. ${ }^{2,4,7,34}$ Furthermore, former investigations demonstrated increased BMP4 expression levels in HCC. ${ }^{14}$ Of note, BMP4 strongly induced hepcidin expression in PHH but HCC cells showed no or only minor induction of hepcidin expression after BMP4 treatment. This again raised the question why hepcidin expression is not induced in HCC cells, where BMP4 is strongly expressed. As BMP4 does not signal via Alk2 and the BMP4-specific receptor subunit Alk3 is not differentially expressed in HCC, we analyzed HJV, a known BMP co-receptor. In contrast to the results of Rapisarda et al, ${ }^{27}$ which demonstrate significant higher HJV expression levels in only one of two HCC cell lines, we were able to show in vitro as well as in vivo that HJV expression is lost in HCC on mRNA and protein level. Further, our data suggest that the loss of HJV is a main cause for the missing effect of BMP4 on hepcidin expression in HCC.

In addition to the BMP signaling cascade, several other pathways are known to be involved in regulation of hepcidin expression. The most important and best characterized are the iron-dependent signaling pathway (through the hereditary hemochromatosis protein called HFE and the TFR2) as well as the inflammatory signaling cascade. Especially inflammatory processes, which are also important for regulation of iron metabolism in cirrhotic liver, ${ }^{35}$ have an important role for the development and progression of HCC. However, up to now the influence of these molecules on hepcidin expression levels in HCC is still unknown. Still and although several studies described enhanced expression levels of inflammatory proteins, in particular IL-6, ${ }^{36-38}$ the data of this study strongly suggest that an intact BMP signaling is essential to mediate an inflammatory response. Furthermore, Mowen et $a l^{39}$ reported that methylthioadenosine (MTA) negatively influence STAT signaling, and we have shown that HCC cells have decreased expression of MTAP (MTA phosphorylase) and as a result higher levels of MTA. ${ }^{40,41}$ Consequently, we revealed a lack of responsiveness of HCC cells to interferon, ${ }^{41}$ and it may be speculated that also signaling of other STAT-mediated ligands like IL- 6 may be impaired in HCC via this mechanism.

Furthermore, a previous publication by Tseng $e t a l^{31}$ did not reveal differences in HFE mRNA levels between tumorous and non-tumorous liver tissues, whereas TFR2 expression was decreased in HCC tissues. In contrast, Kijama et al $l^{30}$ postulated that TFR2 is not differentially expressed. Thus, we analyzed expression levels of TFR2 in HCC cell lines and tissues compared with $\mathrm{PHH}$ and normal liver tissues, respectively. Decreased expression levels of TFR2 in HCC cell lines and tissues were detected, but the impact on hepcidin expression in HCC needs to be determined. This is mainly due to the fact that up to now the mechanism by which TFR2 interacts with the BMP signaling cascade is still unknown. Furthermore, it has to be noted that post-transcriptional regulation of TFR2 expression has been reported, ${ }^{42}$ and one limitation of our study was that we assessed TFR2 expression only at the mRNA level. However, considering that mutation of the HJV gene results in similar effects as mutation of the hepcidin gene (iron accumulation in different organs), which additionally are in strong contrast to the moderate characteristics of the mutated TFR 2 phenotype ${ }^{43}$ we suggest that HJV is the more decisive factor.

To analyze the reasons for loss of HJV expression in HCC, we investigated different possible modulators and identified an AU-rich sequence element in the $3^{\prime}$-untranslated region of $\mathrm{HJV}$ as potential cause for reduced RNA stability and HJV expression in HCC compared with non-tumorous liver.

It may be speculated whether the loss of Alk2 expression and the downregulation of HJV expression have implications beyond iron homeostasis. Thus, besides BMP6 also other BMPs as BMP7 and BMP9 have been reported to signal via Alk2 and herewith affect cancer progression. ${ }^{44,45}$ Future studies have to address whether Alk2-mediated signaling also affects pathophysiology of HCC and whether (loss of) Alk2 may serve as prognostic marker for HCC progression. Moreover, it has to be noted that in most of our experiments we compared normal hepatic tissue and HCC specimens but HCC arises in many cases in cirrhotic or at least diseased livers. Actually, two prior studies reported no significant difference in hepcidin or HJV expression, respectively, between HCC and surrounding non-tumorous livers. ${ }^{31,32}$ It has to be noted that both studies had been performed with tissue specimens from patients with different ethnic background than in our study. Still, it also has to be considered that some of the mechanisms, which we identified in this study as potential explanation for the missing effect of enhanced BMP levels on hepatic hepcidin expression are already affected in pre-malignant diseased liver tissue, and herewith, may affect iron homeostasis in patients with chronic liver disease. Further studies have to address this hypothesis, which might also have therapeutic as well as prognostic implications.

Furthermore, our findings have also to be taken into consideration when using HCC cell lines for the investigation of physiological iron metabolism, since in summary, our study demonstrates deregulation of iron sensing pathways in HCC. In non-tumorous tissue, HJV mediates BMP signalling to enhance hepcidin expression. In HCC, tissue loss of Alk2 and HJV expression is a potential mechanism, which prevents the induction of hepcidin expression, although BMP4 and BMP6 expression levels are enhanced, and herewith avoids a downregulation of iron metabolism in HCC patients.

Supplementary Information accompanies the paper on the Laboratory Investigation website (http://www.laboratoryinvestigation.org) 


\section{ACKNOWLEDGEMENTS}

We are indebted to Thomas Weiss (University Hospital Regensburg, Germany) for providing primary human hepatocytes, and to Rudolf Jung (University Hospital Regensburg, Germany) for excellent technical assistance. Further, we thank Herbert Y Lin (Harvard Medical School, Boston) for providing Flag-tagged human HJV expression plasmid, Gerd Kullak-Ublick (University Hospital Zurich) for providing HNF-1 $\alpha$ expression plasmid and Antonio Garcia de Herreros (University Pompeu Fabra, Barcelona, Spain) for providing antisense snail plasmids. Moreover, we are indebted to Jörg Marienhagen for performing statistical and power analysis. We acknowledge the Human Tissue and Cell Research (HTCR) Foundation for supporting our research by making human liver tissue available. This work was supported by grants from the Medical Faculty of the University of Regensburg (ReForM) to $\mathrm{CH}$ and $\mathrm{AKB}$.

Author contributions: $\mathrm{AKB}$ and $\mathrm{CH}$ designed the study. UM, SA and GK conceived and carried out experiments. All authors analyzed and interpreted data, were involved in writing the paper and had final approval of the submitted and published versions.

\section{DISCLOSURE/CONFLICT OF INTEREST}

The authors declare no conflict of interest.

1. Anderson GJ, Frazer DM, McLaren GD. Iron absorption and metabolism. Curr Opin Gastroenterol 2009;25:129-135.

2. Lin L, Valore EV, Nemeth $\mathrm{E}$, et al. Iron transferrin regulates hepcidin synthesis in primary hepatocyte culture through hemojuvelin and BMP2/4. Blood 2007;110:2182-2189.

3. Babitt JL, Huang FW, Wrighting DM, et al. Bone morphogenetic protein signaling by hemojuvelin regulates hepcidin expression. Nat Genet 2006;38:531-539.

4. Babitt JL, Huang FW, Xia Y, et al. Modulation of bone morphogenetic protein signaling in vivo regulates systemic iron balance. J Clin Invest 2007:117:1933-1939.

5. Xia $\mathrm{Y}$, Babitt $\mathrm{JL}$, Sidis $\mathrm{Y}$, et al. Hemojuvelin regulates hepcidin expression via a selective subset of BMP ligands and receptors independently of neogenin. Blood 2008;111:5195-5204.

6. Andriopoulos Jr B, Corradini E, Xia Y, et al. BMP6 is a key endogenous regulator of hepcidin expression and iron metabolism. Nat Genet 2009:41:482-487.

7. Truksa J, Peng $H$, Lee $P$, et al. Different regulatory elements are required for response of hepcidin to interleukin- 6 and bone morphogenetic proteins 4 and 9. Br J Haematol 2007;139:138-147.

8. Meynard D, Kautz L, Darnaud V, et al. Lack of the bone morphogenetic protein BMP6 induces massive iron overload. Nat Genet 2009;41: 478-481.

9. Kautz L, Meynard D, Monnier A, et al. Iron regulates phosphorylation of Smad1/5/8 and gene expression of Bmp6, Smad7, Id1, and Atoh8 in the mouse liver. Blood 2008;112:1503-1509.

10. Arndt S, Maegdefrau U, Dorn C, et al. Iron-induced expression of BMP6 in intestinal cells is the main regulator of hepatic hepcidin expression in vivo. Gastroenterology 2010;138:372-382.

11. Rothhammer T, Poser I, Soncin F, et al. Bone morphogenic proteins are overexpressed in malignant melanoma and promote cell invasion and migration. Cancer Res 2005;65:448-456.

12. Park $Y$, Kim JW, Kim DS, et al. The bone morphogenesis protein-2 (BMP-2) is associated with progression to metastatic disease in gastric cancer. Cancer Res Treat 2008;40:127-132.

13. Hatakeyama S, Gao YH, Ohara-Nemoto $\mathrm{Y}$, et al. Expression of bone morphogenetic proteins of human neoplastic epithelial cells. Biochem Mol Biol Int 1997;42:497-505.

14. Maegdefrau U, Amann T, Winklmeier A, et al. Bone morphogenetic protein 4 is induced in hepatocellular carcinoma by hypoxia and promotes tumour progression. J Pathol 2009;218:520-529.

15. Blum HE. Hepatocellular carcinoma: therapy and prevention. World $J$ Gastroenterol 2005;11:7391-7400.

16. Mena NP, Esparza A, Tapia V, et al. Hepcidin inhibits apical iron uptake in intestinal cells. Am J Physiol Gastrointest Liver Physiol 2008;294:G192-G198.
17. Nemeth $\mathrm{E}$, Tuttle MS, Powelson J, et al. Hepcidin regulates cellular iron efflux by binding to ferroportin and inducing its internalization. Science 2004;306:2090-2093.

18. Sorrentino $\mathrm{P}, \mathrm{D}^{\prime}$ Angelo $\mathrm{S}$, Ferbo $\mathrm{U}$, et al. Liver iron excess in patients with hepatocellular carcinoma developed on non-alcoholic steatohepatitis. J Hepatol 2009;50:351-357.

19. Hellerbrand C, Poppl A, Hartmann A, et al. HFE C282Y heterozygosity in hepatocellular carcinoma: evidence for an increased prevalence. Clin Gastroenterol Hepatol 2003;1:279-284.

20. Hellerbrand C, Amann T, Schlegel J, et al. The novel gene MIA2 acts as a tumour suppressor in hepatocellular carcinoma. Gut 2008:57:243-251

21. Weiss TS, Jahn B, Cetto $M$, et al. Collagen sandwich culture affects intracellular polyamine levels of human hepatocytes. Cell Prolif 2002;35:257-267.

22. Rothhammer $T$, Bataille $F$, Spruss $T$, et al. Functional implication of BMP4 expression on angiogenesis in malignant melanoma. Oncogene 2007;26:4158-4170.

23. Waxman S, Wurmbach E. De-regulation of common housekeeping genes in hepatocellular carcinoma. BMC Genomics 2007;8:243.

24. Poser I, Dominguez D, de Herreros AG, et al. Loss of E-cadherin expression in melanoma cells involves up-regulation of the transcriptional repressor Snail. J Biol Chem 2001;276:24661-24666.

25. Bosserhoff AK, Moser M, Scholmerich J, et al. Specific expression and regulation of the new melanoma inhibitory activity-related gene MIA2 in hepatocytes. J Biol Chem 2003;278:15225-15231.

26. ten Dijke $\mathrm{P}$, Yamashita $\mathrm{H}$, Sampath $\mathrm{TK}$, et al. Identification of type receptors for osteogenic protein-1 and bone morphogenetic protein4. J Biol Chem 1994:269:16985-16988.

27. Rapisarda C, Puppi J, Hughes RD, et al. Transferrin receptor 2 is crucial for iron sensing in human hepatocytes. Am J Physiol Gastrointest Liver Physiol 2010;299:G778-G783.

28. Wallace DF, Summerville L, Lusby PE, et al. First phenotypic description of transferrin receptor 2 knockout mouse, and the role of hepcidin. Gut 2005;54:980-986.

29. Sugimachi K, Tanaka S, Kameyama T, et al. Transcriptional repressor snail and progression of human hepatocellular carcinoma. Clin Cancer Res 2003;9:2657-2664.

30. Kijima H, Sawada T, Tomosugi $\mathrm{N}$, et al. Expression of hepcidin mRNA is uniformly suppressed in hepatocellular carcinoma. BMC Cancer 2008;8:167.

31. Tseng HH, Chang JG, Hwang $\mathrm{YH}$, et al. Expression of hepcidin and other iron-regulatory genes in human hepatocellular carcinoma and its clinical implications. J Cancer Res Clin Oncol 2009:135:1413-1420.

32. Tan MG, Kumarasinghe MP, Wang SM, et al. Modulation of iron-regulatory genes in human hepatocellular carcinoma and its physiological consequences. Exp Biol Med (Maywood) 2009;234: 693-702.

33. Rosenzweig BL, Imamura $\mathrm{T}$, Okadome $\mathrm{T}$, et al. Cloning and characterization of a human type II receptor for bone morphogenetic proteins. Proc Natl Acad Sci USA 1995;92:7632-7636.

34. Zhang AS, Yang F, Wang J, et al. Hemojuvelin-neogenin interaction is required for bone morphogenic protein-4-induced hepcidin expression. J Biol Chem 2009;284:22580-22589.

35. Sheikh N, Batusic DS, Dudas J, et al. Hepcidin and hemojuvelin gene expression in rat liver damage: in vivo and in vitro studies. Am J Physiol Gastrointest Liver Physiol 2006;291:G482-G490.

36. Soresi M, Giannitrapani L, D'Antona F, et al. Interleukin-6 and its soluble receptor in patients with liver cirrhosis and hepatocellular carcinoma. World J Gastroenterol 2006;12:2563-2568.

37. Moran DM, Mattocks MA, Cahill PA, et al. Interleukin-6 mediates $\mathrm{G}(0)$ / $G(1)$ growth arrest in hepatocellular carcinoma through a STAT 3-dependent pathway. J Surg Res 2008;147:23-33.

38. Porta C, De Amici M, Quaglini S, et al. Circulating interleukin-6 as a tumor marker for hepatocellular carcinoma. Ann Oncol 2008;19:353-358.

39. Mowen KA, Tang J, Zhu W, et al. Arginine methylation of STAT1 modulates IFNalpha/beta-induced transcription. Cell 2001;104:731-741.

40. Kirovski G, Stevens AP, Czech B, et al. Down-regulation of methylthioadenosine phosphorylase (MTAP) induces progression of hepatocellular carcinoma via accumulation of $5^{\prime}$-deoxy-5'methylthioadenosine (MTA). Am J Pathol 2011;178:1145-1152. 
41. Hellerbrand $\mathrm{C}$, Muhlbauer $\mathrm{M}$, Wallner $\mathrm{S}$, et al. Promoterhypermethylation is causing functional relevant downregulation of methylthioadenosine phosphorylase (MTAP) expression in hepatocellular carcinoma. Carcinogenesis 2006;27:64-72.

42. Johnson MB, Enns CA. Diferric transferrin regulates transferrin receptor 2 protein stability. Blood 2004;104:4287-4293.

43. Chen J, Chloupkova M. Abnormal iron uptake and liver cancer. Cancer Biol Ther 2009;8:1699-1708.
44. Herrera $B$, van Dinther $M$, ten Dijke $P$, et al. Autocrine bone morphogenetic protein-9 signals through activin receptor-like kinase-2/Smad1/Smad4 to promote ovarian cancer cell proliferation. Cancer Res 2009;69:9254-9262.

45. $\mathrm{Na} \mathrm{YR}$, Seok SH, Kim DJ, et al. Bone morphogenetic protein 7 induces mesenchymal-to-epithelial transition in melanoma cells, leading to inhibition of metastasis. Cancer Sci 2009;100: 2218-2225. 